\title{
Radiotherapy or Chemoradiation in Unresectable Biliary Cancer: A Retrospective Study
}

\author{
SILVIA BISELLO ${ }^{1}$, MILLY BUWENGE ${ }^{1}$, ANDREA PALLONI ${ }^{2}$, ROSA AUTORINO ${ }^{3}$, \\ FRANCESCO CELLINI ${ }^{3}$, GABRIELLA MACCHIA ${ }^{4}$, FRANCESCO DEODATO ${ }^{4}$, SAVINO CILLA $^{5}$, \\ GIOVANNI BRANDI ${ }^{2}$, LUCA TAGLIAFERRI ${ }^{3}$, SILVIA CAMMELLI ${ }^{1}$, \\ VINCENZO VALENTINI $^{3}$, ALESSIO G. MORGANTI ${ }^{1 *}$ and GIAN C. MATTIUCCI ${ }^{3 *}$ \\ ${ }^{1}$ Radiation Oncology Center, Department of Experimental, Diagnostic and Speciality Medicine-DIMES, \\ University of Bologna, S. Orsola-Malpighi Hospital, Bologna, Italy; \\ ${ }^{2}$ Department of Medical and Surgical Sciences - DIMEC, University of Bologna, \\ S. Orsola-Malpighi Hospital, Bologna, Italy; \\ ${ }^{3}$ Fondazione Policlinico Universitario "A. Gemelli" IRCCS, Rome, Italy; \\ ${ }^{4}$ Radiation Oncology Unit and ${ }^{5}$ Medical Physics Unit, Fondazione "Giovanni Paolo II", \\ Catholic University of the Sacred Heart, Campobasso, Italy
}

\begin{abstract}
Background/Aim: To retrospectively evaluate the outcome of patients with unresectable biliary cholangiocarcinoma $(C C)$ treated with radiotherapy $(R T)$ plus/minus chemotherapy (CHT). Materials and Methods: Data of patients with intrahepatic CC (ICC), Klatskin's tumor (KT), distal extrahepatic CC (ECC), and gallbladder cancer $(G B C)$ diagnosed from 1991 to 2017 were retrospectively analyzed. The treatment was mainly based on RT plus concurrent CHT +/- brachytherapy (BRT) boost. The Kaplan-Meier method was used to calculate survival curves that were compared using the log-rank test. Results: Seventy-six patients were included in this analysis (males: 59\%; females: 41\%; median age: 66.5 years). A minority of patients $(7.9 \%)$ were treated for disease recurrence after surgery. According to TNM, $78.5 \%$ of patients had T stage $>3$ and $77.6 \%$ of patients were treated with concurrent CHT$R T$ while $22.3 \%$ received $R T$ followed by sequential $C H T$. Median RT dose was 50 Gy (range: 16-75 Gy) delivered with conventional fractionation. CHT was based on Gemcitabine or 5-fluorouracil. BRT was prescribed to $51.3 \%$ of patient
\end{abstract}

\footnotetext{
*These Authors contributed equally to this study.

Correspondence to: Milly Buwenge, MSc, Radiation Oncology Center, Department of Experimental, Diagnostic and Specialty Medicine - DIMES, University of Bologna, S. Orsola-Malpighi Hospital, via Giuseppe Massarenti 9, 40138 Bologna, Italy. Tel: +39 0512143564, Fax: +39 0516364336, e-mail: mbuwenge@gmail.com

Key Words: Radiotherapy, chemoradiation, biliary tract cancers, cholangiocarcinoma.
}

with a median dose of 14 Gy. Reported Grade $\geq 3$ acute GI and hematological toxicity were $13.2 \%$ and $8.1 \%$, respectively. No other severe acute toxicities were reported. One- and 2-year overall survival (OS) were $58.1 \%$ and $25.8 \%$, respectively (median: 13.5 months), while 1 - and 2year progression-free survival (PFS) were $43.4 \%$ and $9.4 \%$, respectively. None of the following variables had a significant impact on OS and PFS: BRT boost, tumor site, concurrent CHT, and the drugs used in concurrent CHT. In contrast, patients receiving $R T$ with $2 D$ technique showed a PFS significantly higher compared to patients treated with the 3D technique (median: 15.5 vs. 8.5 months; $p=0.02$ ). Conclusion: Combined modality treatment $(R T+C H T \pm B R T)$ in unresectable biliary cancer was associated with acceptable toxicity and OS comparable to the actual standard treatment $(C H T)$. The significantly improved PFS in patients undergoing $2 D-R T$ raises doubts regarding the adequacy of target delineation in these neoplasms.

Biliary tract cancers (BTC) are aggressive malignancies that are rare in the western world, but largely diffused in many Asian countries. BTC are commonly classified into intrahepatic cholangiocarcinoma (ICC), Klatskin's tumor (KT), extrahepatic cholangiocarcinoma (ECC), and gallbladder cancer (GBC) (1). Surgery, when feasible, represents the standard of care and in some cases is followed by adjuvant chemotherapy (CHT) or chemoradiation (CHTRT). However, the most common presentation of these tumors is locally advanced stage with a life expectancy of few months $(2,3)$.

The treatment of unresectable disease is challenging due to patients' old age and the aggressive nature of BTC. The standard treatment is CHT, with radiotherapy (RT) or 
chemoradiation (CHTRT) +/- brachytherapy (BT) boost considered as an alternative option $(1,3)$.

There is no standard CHT regimen for the advanced stages of disease (locally advanced and metastatic), although the NCCN guidelines suggest the combination of gemcitabine and oxaliplatin (3). A pooled analysis (4) reported median overall survival (OS) of 8.2 months with a positive trend on OS in patients treated with drugs combination (9.3 months vs. 7.5 months, $p$-value $=0.061$ ).

Considering only locally advanced non-metastatic patients, a recent paper reported outcomes in patients treated with gemcitabine plus cisplatin CHT with 13.8- and 6.7-months median OS and progression-free survival (PFS), respectively (5). Several studies in the literature have reported similar survival rates in patients treated with combined modality treatment based on CHTRT. Yi and coworkers (6) reported 42.6 weeks median OS using concurrent CHTRT based on 5-FU or gemcitabine and a total RT dose of $50.4 \mathrm{~Gy}$. Lee and colleagues (7) who prescribed RT $(55.1 \mathrm{~Gy})$ plus concurrent 5-FU, reported a median OS of 13.5 months. Moreover, some studies (8-13) have suggested a potential role of BRT boost in improving OS. Particularly, in the series of Deodato and colleagues and Foo and coworkers, some of the patients treated with BRT boost survived for over 5 years $(16.7 \%$ and $14.1 \%$, respectively) $(8,11)$.

It is worth noting that sample sizes of most studies were small and consequently their results should be interpreted with caution. Therefore, the aim of this study was to perform a retrospective analysis of a relatively large series of patients with unresectable BTC treated with RT/CHTRT +/- BRT boost.

\section{Materials and Methods}

Study design and eligibility criteria. We retrospectively collected data of patients treated from 1991 to 2017. Endpoints of the study were to analyze OS, PFS, acute toxicity, and the prognostic impact of several disease and treatment related parameters. All patients had a previous diagnosis of unresectable BTC (ICC, KT, ECC, or GBC). Most of them received concurrent CHTRT eventually followed by BRT boost. Patients who underwent surgery or palliative BRT alone were excluded. The diagnosis of unresectable disease was based on imaging (CT scan or MRI) or surgical exploration. This analysis was approved by the local Ethical Board. Only patients who gave their written informed consent for the scientific use of their data were included in this analysis.

Treatment characteristics. Patients underwent 2D-standard RT or 3Dconformal RT. In patients treated with 2D-RT, a three-field technique (one anterior and two opposed lateral rectangular fields) was used. The fields were designed to adequately cover the tumor volume (with a margin of at least $2.0-2.5 \mathrm{~cm}$ ) and the primary lymphatic drainage (ICC and KT: hilar nodes along hepatic artery; GB and ECC: hilar and hepatic artery nodes, celiac, peripancreatic, superior mesenteric and para-aortic area nodes). The beam dimensions were shaped based on the diagnostic computed tomography (CT) scans findings. Patients treated with 3D-RT were immobilized in a customized Alpha Cradle and underwent a CT simulation. The gross tumor volume (GTV) was defined as the macroscopic mass visible
Table I. Treatment characteristics.

\begin{tabular}{lc}
\hline Treatment performed & Value $(\%)$ \\
\hline RT + BRT & $15(19.7)$ \\
RT + concurrent gemcitabine & $27(35.5)$ \\
RT + concurrent gemcitabine + BRT & $9(11.8)$ \\
RT + concurrent 5-Fluorouracil & $6(7.9)$ \\
RT + concurrent 5-Fluorouracil + BRT & $12(15.8)$ \\
RT + concurrent gemcitabine and oxaliplatin & $2(2.6)$ \\
RT + concurrent gemcitabine and oxaliplatin + BRT & $2(2.6)$ \\
RT + concurrent capecitabine + BRT & $1(1.3)$ \\
RT + sequential capecitabine & $1(1.3)$ \\
RT + sequential gemcitabine and cisplatin & $1(1.3)$ \\
RT & \\
Median dose (range), Gy & $50(16-75)$ \\
Fractionation, cGy & 180 \\
\hline Planning target volume expansion & CTV +1 cm \\
& radially \\
\hline BRT & $39(51.3)$ \\
Yes & $37(48.7)$ \\
No & $14(14-50)$ \\
Median dose (range), Gy &
\end{tabular}

BRT: Brachytherapy; CTV: clinical target volume; RT: radiotherapy.

Table II. Acute toxicity (Radiation Therapy Oncology Group scale).

\begin{tabular}{lcrrrr}
\hline Toxicity & \multicolumn{5}{c}{ Grade (\%) } \\
\cline { 2 - 6 } & \multicolumn{1}{c}{0} & \multicolumn{1}{c}{1} & \multicolumn{1}{c}{2} & \multicolumn{1}{l}{3} & \multicolumn{1}{c}{4} \\
\hline Gastrointestinal & $28(41.8)$ & $20(29.8)$ & $11(16.4)$ & $6(9.0)$ & $2(3.0)$ \\
Hematological & $33(53.2)$ & $16(25.8)$ & $8(12.9)$ & $4(6.5)$ & $1(1.6)$ \\
\hline
\end{tabular}

on CT images while the clinical target volume (CTV) included GTV plus $1 \mathrm{~cm}$ isotropic margin and nodes with high risk of microscopic disease (same as for 2D technique). The planning target volume (PTV) was defined by adding an isotropic $1 \mathrm{~cm}$ margin to the CTV.

Patients' first follow up visit was scheduled at 3 months after the end of RT and subsequently every 6 months for 5 years and then annually. At each visit both laboratory tests and chest-abdominal CT scans were evaluated.

Statistical analysis. Descriptive values (median and range for continuous variables and frequencies and percentages for categorical variables) were calculated. Toxicity was scored according to the Radiation Therapy Oncology Group criteria (RTOG) (14). Only acute toxicity defined as any adverse event occurring within 3 months from the end of RT is reported in this analysis.

PFS was calculated from the date of diagnosis to the date of discovery of any (local or systemic) disease recurrence or last follow-up. OS was calculated from the date of diagnosis to the date of death or last follow up. Actuarial PFS and OS were calculated with the Kaplan-Meyer method (15) and the differences between 


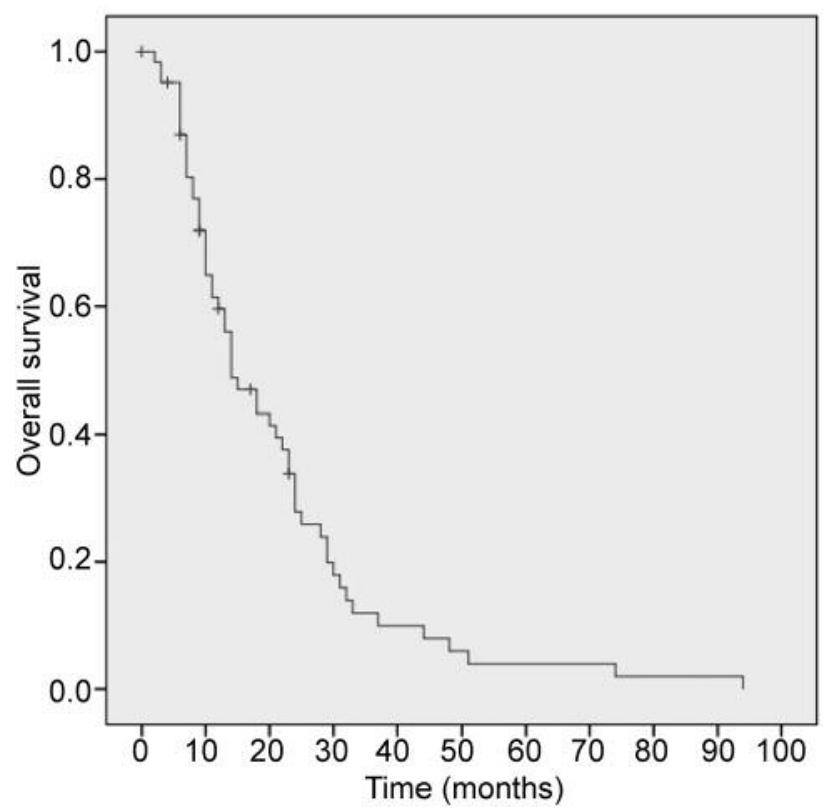

Figure 1. Actuarial overall survival (all patients).

survival curves were compared with the log-rank test (16). Statistical analysis was performed with IBM SPSS (IBM SPSS Statistics for Windows, Inc, Version 20.0; IBM Corp, Armonk, NY, USA).

\section{Results}

A total of 76 patients, were included in this analysis (45 males and 31 females). Median age was 66.5 years (range $=33-88$ years). All patients had a diagnosis of unresectable BTC and were classified according to the location of the disease (ICC: 3.9\%; KT: $51.3 \%$; ECC: $32.9 \%$, GBC: $3.9 \%$ ) and $7.8 \%$ were treated for local relapse after surgery. Metastatic regional nodes were detected in $36.8 \%$ of patients.

Median RT dose was 50 Gy delivered with conventional fractionation. Concurrent CHT was prescribed to $77.6 \%$ of patients and was based on Gemcitabine or 5-Fluorouracil while the remaining received RT alone (19.7\%) or RT followed by adjuvant CHT (2.6\%). A sequential low or high dose-rate BRT boost (median dose of $14 \mathrm{~Gy}$ ) was delivered using ${ }^{192}$ Iridium sources to $51.3 \%$ of patients. Table I shows all the treatment combinations.

Acute toxicity is reported in Table II. The incidence of grade $\geq 3$ gastrointestinal and hematological acute toxicity was $13.2 \%$ and $8.1 \%$, respectively. Four patients showed acute cholangitis with a fever $>38.0^{\circ} \mathrm{C}$.

One-, 2-, and 3-year OS were $58.1 \%, 25.8 \%$, and $11.2 \%$, respectively (Figure 1). One-, 2-, and 3-year PFS were $43.4 \%, 9.4 \%$, and $9.4 \%$, respectively (Figure 2). Median OS and PFS were 13.5 and 10.0 months, respectively.

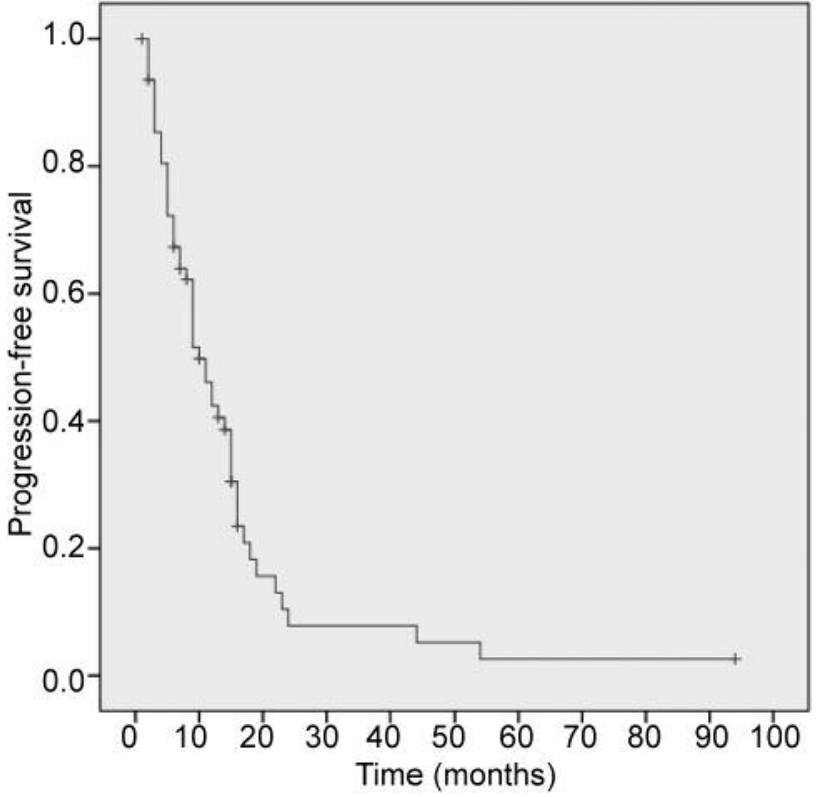

Figure 2. Actuarial progression-free survival (all patients).

Table III shows the results of the univariate analysis. Analyzing the impact of BRT boost on OS, no statistical difference was found $(p=0.61)$ but a higher rate of longterm survivors was recorded in the BRT group. In fact, 2year OS was $21.4 \%$ in the BRT boost group and $30.8 \%$ for patients not receiving BRT boost, while 4-year OS was $12.2 \%$ in the BRT boost group and $0.0 \%$ in the other patients (Figure 3).

Moreover, no significant differences were recorded both in terms of OS and PFS between the different BTC sites or nodal involvement. Similarly, we did not observe significant differences in terms of outcome among patients undergoing either RT alone or concurrent CHTRT.

Considering the RT technique, no statistical difference was found in terms of OS for the group of patients treated with 2D and the ones treated with 3D-RT (median OS, 2D-RT: 22.0 months versus 3D-RT: 13.5 months; $p=0.13$ ), while a statistically significant difference in terms of PFS was recorded (median PFS: 2D-RT: 15.5 months, 3D-RT: 8.5 months; $p=0.02$ ) (Figure 4). Finally, no statistical differences in terms of OS and PFS were found between gemcitabineand 5-fluorouracil-based CHTRT.

\section{Discussion}

To the best of our knowledge, this is one of the largest series of patients treated with RT for unresectable BTC. The aim of the study was to evaluate the efficacy of RT +/- CHT +/BRT boost. 


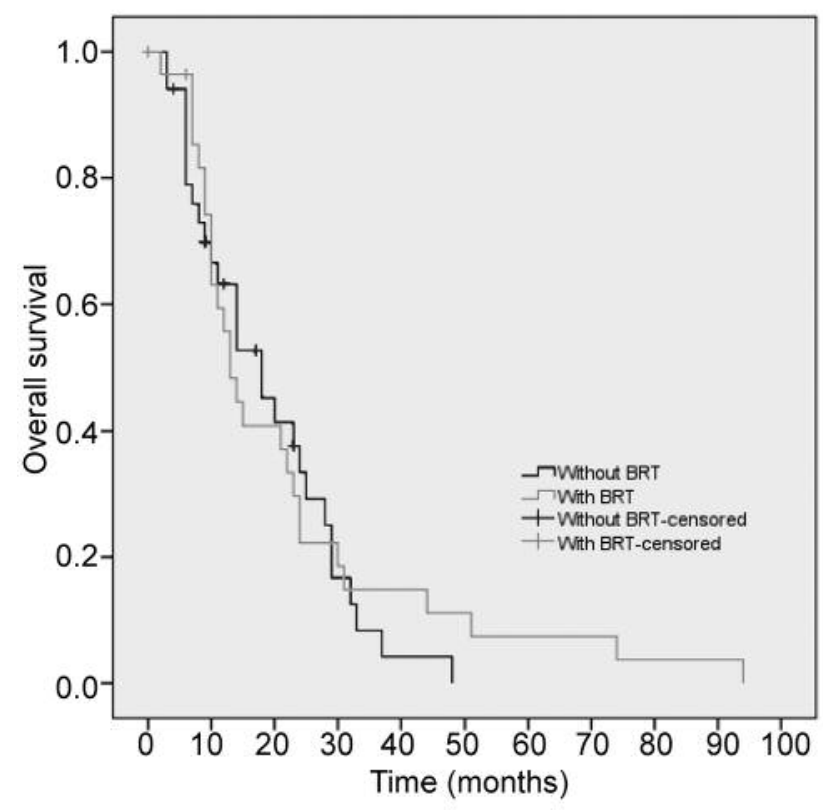

Figure 3. Overall survival: patients treated with chemoradiation plus brachytherapy (grey line) versus chemoradiation without brachytherapy (black line).

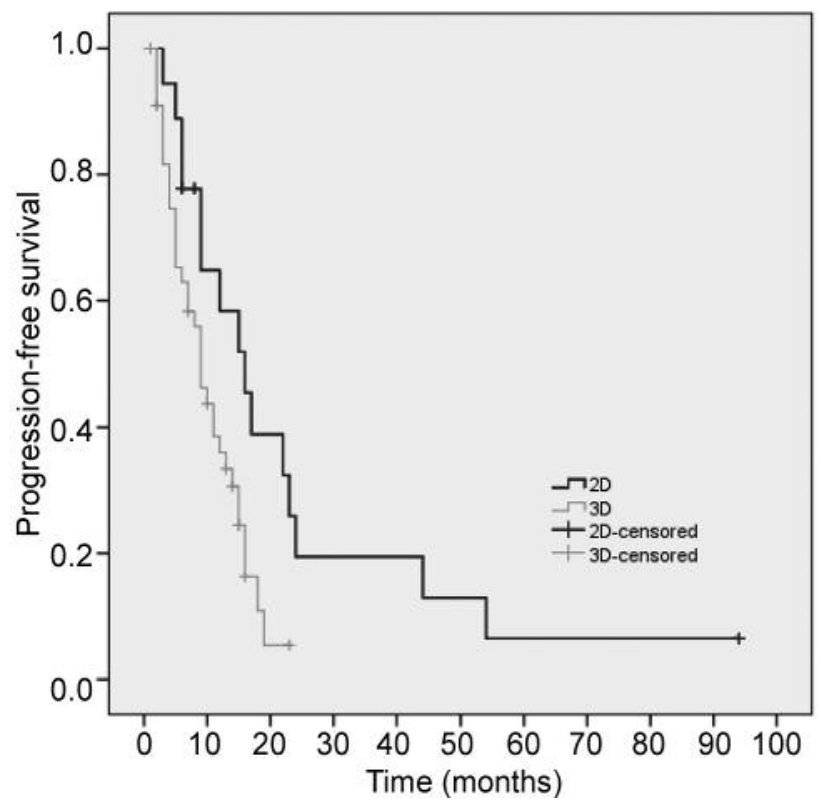

Figure 4. Progression-free survival: patients treated with $2 D$ technique (black line) versus $3 D$ technique (grey line).

Table III. Univariable analysis including 1-, 2- and 3-year overall survival, progression-free survival and log rank p-value.

\begin{tabular}{|c|c|c|c|c|c|c|c|c|c|c|c|}
\hline & \multirow[b]{2}{*}{$\begin{array}{l}\mathrm{N}^{\circ} \\
\text { pts }\end{array}$} & \multicolumn{5}{|c|}{ OS } & \multicolumn{5}{|c|}{ PFS } \\
\hline & & $\begin{array}{l}\text { Median } \\
\text { (months) }\end{array}$ & $\begin{array}{c}\text { 1-year } \\
(\%)\end{array}$ & $\begin{array}{c}\text { 2-year } \\
(\%)\end{array}$ & $\begin{array}{c}\text { 3-year } \\
(\%)\end{array}$ & $p$-Value & $\begin{array}{l}\text { Median } \\
\text { (months) }\end{array}$ & $\begin{array}{c}1 \text {-year } \\
(\%)\end{array}$ & $\begin{array}{c}2 \text {-year } \\
(\%)\end{array}$ & $\begin{array}{c}\text { 3-year } \\
(\%)\end{array}$ & $p$-Value \\
\hline All population & 76 & 13.5 & 58.1 & 25.8 & 11.2 & & 10 & 43.4 & 9.4 & 9.4 & \\
\hline \multicolumn{12}{|c|}{ Brachytherapy boost } \\
\hline Yes & 39 & 12.5 & 51.9 & 21.4 & 12.2 & \multirow[t]{2}{*}{0.61} & 13.5 & 50.6 & 13.2 & 13.2 & \multirow[t]{2}{*}{0.34} \\
\hline No & 37 & 14.5 & 64.4 & 30.8 & 0.0 & & 9.0 & 37.9 & 8.0 & 8.0 & \\
\hline \multicolumn{12}{|l|}{ Tumor site } \\
\hline ICC & 3 & 9.5 & 0.0 & 0.0 & 0.0 & \multirow{5}{*}{0.43} & 4.0 & 0.0 & 0.0 & 0.0 & \multirow{5}{*}{0.11} \\
\hline $\mathrm{KT}$ & 39 & 13.5 & 55.0 & 23.8 & 10.2 & & 9.5 & 39.1 & 11.4 & 11.4 & \\
\hline ECC & 25 & 18.0 & 64.0 & 29.9 & 12.8 & & 12.5 & 51.3 & 15.6 & 15.6 & \\
\hline GBC & 3 & 18.0 & 66.7 & 0.0 & 0.0 & & 15.0 & 66.7 & 0.0 & 0.0 & \\
\hline Relapse & 6 & 10.0 & 66.7 & 0.0 & 0.0 & & 9.0 & 33.3 & 0.0 & 0.0 & \\
\hline \multicolumn{12}{|c|}{ Nodal involvement } \\
\hline Negative & 48 & 13.5 & 59.0 & 27.5 & 16.5 & \multirow[t]{2}{*}{0.27} & 10.5 & 45.9 & 18.3 & 18.3 & \multirow[t]{2}{*}{0.09} \\
\hline Positive & 28 & 12.5 & 56.5 & 22.7 & 0.0 & & 9.5 & 39.1 & 0.0 & 0.0 & \\
\hline \multicolumn{12}{|l|}{ RT technique } \\
\hline $2 \mathrm{D}$ & 19 & 22.0 & 61.1 & 33.3 & 22.2 & \multirow[t]{2}{*}{0.13} & 15.5 & 58.3 & 19.4 & 19.4 & \multirow[t]{2}{*}{0.02} \\
\hline $3 \mathrm{D}$ & 57 & 13.5 & 56.4 & 23.7 & 6.0 & & 8.5 & 36.0 & 5.4 & 0.0 & \\
\hline CHTRT & 61 & 13.5 & 60.1 & 24.4 & 11.2 & \multirow[t]{2}{*}{0.71} & 10.5 & 44.1 & 9.9 & 9.9 & \multirow[t]{2}{*}{0.21} \\
\hline RT & 15 & 9.5 & 48.9 & 32.6 & 10.9 & & 6.0 & 38.1 & 0.0 & 0.0 & \\
\hline \multicolumn{12}{|l|}{ Chemotherapy } \\
\hline 5-Fluorouracil & 18 & 13.0 & 55.6 & 27.8 & 16.7 & \multirow[t]{2}{*}{0.51} & 13.5 & 55.6 & 13.9 & 13.9 & \multirow[t]{2}{*}{0.14} \\
\hline Gemcitabine & 34 & 12.5 & 61.4 & 27.2 & 9.1 & & 9.5 & 38.6 & 12.1 & 12.1 & \\
\hline
\end{tabular}

CHTRT: Chemoradiation; ECC: extrahepatic cholangiocarcinoma; GBC: gallbladder cancer; GI: gastrointestinal; ICC: intrahepatic cholangiocarcinoma; KT: Klatskin's tumor; $\mathrm{N}^{\circ}$ pts: number of patients; OS: overall survival, PFS: progression-free survival; RT: radiotherapy; 2D; two-dimensional; 3D: three-dimensional. 
This analysis has certain limitations due to the retrospective design of the study; the large inhomogeneity in terms of tumor sub-sites and treatments, lack of late toxicity evaluation, and a prolonged period of patients' inclusion with obvious consequent differences in terms of staging and treatments techniques. However, the present study analyzed a much larger sample of patients compared to other recent studies (17).

In our experience, treatment was generally well-tolerated with $12.1 \%$ and $8.2 \%$ grade $\geq 3$ gastrointestinal and hematological toxicity rates, respectively.

According to recent guidelines (1), there is no standard therapy for locally advanced BTC. In this setting, Kim and colleagues (5) reported 13.8- and 6.7-months median OS and PFS, respectively, in patients who were treated with first-line gemcitabine and cisplatin. Ji and coworkers (18) reported a median OS of 16.7 months in patients treated with CHT who were included in their multi-institutional propensity score matching analysis. The results achieved in these studies are not clearly different from those of our analysis (median OS and PFS: 13.5 and 10 months, respectively).

The results of the present report are similar to the ones of our recent review on 6 studies published in the last ten years on combined CHTRT in unresectable BTC. In fact, the median values from the 6 included papers were 13.0 months and 7.5 months median OS and PFS, respectively (17).

In our series, in a sub analysis on the RT techniques used, a statistical difference was found in terms of PFS between patients treated with 2D-RT and the ones treated with $3 \mathrm{D}$ technique with an improved PFS in the first group ( $p=0.02)$ (Figure 4$)$. This result can be interpreted as a sign of an inappropriate target definition in patients receiving 3D-RT. Therefore, the use of guidelines for CTV contouring in these patients could be useful (19).

OS was comparable between the different sites of disease perhaps due to the small sample size of some subgroups. However, it can be observed that the two patients with longer survival (74 and 94 months) had ECC and underwent BRT boost.

BRT boost has been used in several centers to improve local control and potentially OS. In our experience OS was not improved in patients who underwent BRT boost. However, we recorded a higher rate of long-term survivors ( $>48$ months) in the BRT group (Figure 3). This data confirms a previous analysis reporting $16.7 \%$ and $0 \% 5$-year OS in patients treated with and without BRT boost, respectively (8).

Stereotactic RT represents an emerging technique in RT and has been used in some studies on locally advanced BTC. A recent review of the literature showed that in terms of OS (median: 15 months) the results are similar to those recorded in the present analysis with CHTRT and that treatment is reasonably tolerated (20). Furthermore, this technique has an advantage given its brevity of an easy integration with systemic therapy.
In conclusion, CHTRT results in the treatment of locally advanced BTC seem similar to those of standard CHT. Therefore, this combined modality treatment can still represent a therapeutic option in this setting. Future studies are necessary to improve clinical outcomes. These studies could be aimed at: i) comparing standard CHTRT to stereotactic RT, ii) identifying new ways of integration of CHTRT and systemic therapies, iii) identifying predictive factors of the effectiveness of different treatments to allow a personalized choice between them (CHT or CHTRT or stereotactic RT), iv) assessing the impact of standardized methods in target definition.

Considering that the prognosis remains unfavorable in these patients, the main aim of treatment is palliation. Therefore, in future trials it would be important to perform a detailed analysis of treatment impact on patients' quality of life.

\section{Acknowledgements}

None.

\section{Conflicts of Interest}

The Authors have no actual or potential conflicts of interest regarding this paper.

\section{Authors' Contributions}

Conception and design: AGM, SB, MB, VV, and GCM; Data collection: SB, AP, RA, SiC, LT, GM and FD; Analysis and interpretation of data: $\mathrm{GB}, \mathrm{MB}, \mathrm{SB}, \mathrm{FC}$ and $\mathrm{SaC}$; Manuscript writing: AGM, SB, MB, GB and GCM. All Authors read and approved the final manuscript and gave consent to publication.

\section{References}

1 Valle JW, Borbath I, Khan SA, Huguet F, Gruenberger T, Arnold D and ESMO Guidelines Committee: Biliary cancer: ESMO Clinical Practise Guidelines for diagnosis, treatment and follow up. Annal Oncol 5: v28-v37, 2016. PMID: 27664259. DOI: 10.1093/annonc/mdw324

2 Park J, Kim MH, Kim KP, Park DH, Moon SH, Song TJ, Eum J, Lee SS, Seo DW and Lee SK: Natural history and prognostic factors of advanced cholangiocarcinoma without surgery, chemotherapy, or radiotherapy: A large-scale observational study. Gut Liver 3: 298-305, 2009. PMID: 20431764. DOI: 10.5009/gnl.2009.3.4.298

3 Sharma A, Dwary AD, Mohanti BK, Deo SV, Pal S, Sreenivas V, Raina V, Shukla NK, Thulkar S, Garg P and Chaudhary SP: Best supportive care compared with chemotherapy for unresectable gall bladder cancer: A randomized controlled study. J Clin Oncol 28: 4581-4586, 2010. PMID: 20855823. DOI: 10.1200/JCO.2010.29.3605

4 Eckel F and Schmid RM: Chemotherapy in advanced biliary tract carcinoma: a pooled analysis of clinical trials. $\mathrm{Br} \mathrm{J}$ Cancer 96: 896-902, 2007. PMID: 17325704. 
5 Kim BJ, Hyung J, Yoo C, Kim KP, Park SJ, Lee SS, Park DH, Song TJ, Seo DW, Lee SK, Kim MH, Park JH, Cho H, Ryoo BY and Chang HM: Prognostic factors in patients with advanced biliary tract cancer treated with first-line gemcitabine plus cisplatin: retrospective analysis of 740 patients. Cancer Chemother Pharmacol 80: 209-215, 2017. PMID: 28597043. DOI: $10.1007 / \mathrm{s} 00280-017-3353-2$

6 Yi SW, Kang DR, Kim KS, Park MS, Seong J, Park JY, Bang SM, Song SY, Chung JB and Park SW: Efficacy of concurrent chemoradiotherapy with 5-fluorouracil or gemcitabine in locally advanced biliary tract cancer. Cancer Chemother Pharmacol 73: 191-198, 2014. PMID: 24322374. DOI: 10.1007/s00280-0132340-5

7 Lee KJ, Yi SW, Cha J, Seong J, Bang S, Song SY, Kim HM and Park SW: A pilot study of concurrent chemoradiotherapy with gemcitabine and cisplatin in patients with locally advanced biliary tract cancer. Chemoter Pharmacol 78: 841-846, 2016. PMID: 27586966. DOI: 10.1007/s00280-016-3143-2

8 Deodato F, Clemente G, Mattiucci GC, Macchia G, Costamagna G, Giuliante F, Smaniotto D, Luzi S, Valentini V, Mutignani M, Nuzzo G, Cellini N and Morganti AG: Chemoradiation and brachytherapy in biliary tract carcinoma: Long-term results. Int J Radiat Oncol Biol Phys 64: 483-488, 2006. PMID: 16242254.

9 Autorino R, Mattiucci GC, Ardito F, Balducci M, Deodato F, Macchia G, Mantini G, Perri V, Tringali A, Gambacorta MA, Tagliaferri L, Giuliante F, Morganti AG and Valentini V: Radiochemotherapy with gemcitabine in unresectable extrahepatic cholangiocarcinoma: Long term results of a phase II study. Anticancer Res 36: 737-740, 2016. PMID: 26851032.

10 Mattiucci GC, Autorino R, D'Agostino GR, Deodato F, Macchia G, Perri V, Tringali A, Morganti AG, Mutignani M and Valentini V: Chemoradiation and Brachiterapy in extrahepatic bile duct carcinoma. Crit Rev Onc Hemat 90: 58-67, 2014. PMID: 24289902. DOI: 10.1016/j.critrevonc.2013.10.007

11 Foo ML, Gunderson LL and Bender CE: External radiation therapy and transcatheter iridium in the treatment of extrahepaticbile duct carcinoma. Int J Radiat Oncol Biol Phys 39: 929-935, 1997. PMID: 9369143.

12 Brunner TB, Schwab D and Meyer T: Chemoradiation may prolong survival of patients with non-bulky unresectable extrahepatic biliary carcinoma.Strahlenther Onkol 180: 751-757, 2004. PMID: 15592694.

13 Morganti AG, Trodella L, Valentini V, Montemaggi P, Costamagna G, Smaniotto D, Luzi S, Ziccarelli P, Macchia G, Perri V, Mutignani $\mathrm{M}$ and Cellini N: Combined modality treatment in unresectable, extrahepatic biliary carcinoma. Int J Radiat Oncol 46: 913-919, 2000. DOI: 10.1016/S0360-3016(99) 00487-3
14 Cox JD, Stetz J and Pajak TF: Toxicity criteria of the Radiation Therapy Oncology Group (RTOG) and the European Organization for Research and Treatment of Cancer (EORTC). Int J Radiat Oncol Biol Phys 31: 1341-1346, 1995. DOI: 10.1016/03603016(95)00060-C

15 Kaplan FL and Meier P: Nonparametric estimation from incomplete observations. Am J Stat Assoc 53: 457-481, 1958. DOI: $10.2307 / 2281868$

16 Peto $\mathrm{R}$ and Peto J: Asymptotically efficient rank invariant procedures. J R Stat Soc 135: 185-207, 1972. DOI: 10.2307/ 2344317

17 Bisello S, Buwenge M, Zamagni A, Deodato F, Macchia G, Alessandra A, Cammelli S, Mattiucci GC, Cellini F and Morganti AG: Chemoradiation in unresectable biliary tract cancer: A systematic review. J Gastrointest Oncol, 2018. DOI: 10.21037/jgo.2018.07.10

18 Ji JH, Kim YS, Park I, Lee SI, Kim RB, Park JO, Oh SY, Hwang IG, Jang JS, Song HN and Kang JH: Chemotherapy versus best supportive care in advanced biliary tract carcinoma: A multiinstitutional propensity score matching analysis. Cancer Res Treat 50: 791-800, 2018. PMID: 28838033. DOI: 10.4143/ crt.2017.044

19 Bisello S, Renzulli M, Buwenge M, Calculli L, Sallustio G, Macchia G, Deodato F4, Mattiucci G, Cammelli S, Arcelli A, Giaccherini L, Cellini F, Brandi G, Guerri S, Cilla S, Golfieri R, Fuccio L, Morganti AG and Guido A: An atlas for clinical target volume definition, including elective nodal irradiation in definitive radiotherapy of biliary cancer. Oncol Lett 17: 17841790, 2019. PMID: 30675238. DOI: 10.3892/ol.2018.9774

20 Frakulli R, Buwenge M, Macchia G, Cammelli S, Deodato F, Cilla S, Cellini F, Mattiucci GC, Bisello S, Brandi G, Parisi S and Morganti AG. Stereotactic body radiation therapy in cholangiocarcinoma: a systematic review. Br J Radiol 92(1097): 20180688, 2019. PMID: 30673295. DOI: 10.1259/bjr.20180688

Received March 25, 2019

Revised May 14, 2019

Accepted May 15, 2019 